\title{
Correction to: Systematic review of randomized controlled trials for chronic fatigue syndrome/myalgic encephalomyelitis (CFS/ME)
}

\author{
Do-Young Kim', Jin-Seok Lee², Samuel-Young Park' , Soo-Jin Kim ${ }^{1}$ and Chang-Gue Son ${ }^{2 *}$
}

\section{Correction to: J Transl Med (2020) 18:7.}

https://doi.org/10.1186/s12967-019-02196-9

Following publication of the original article [1], the authors noted that one study (PACE trial) [2] had been missed in the captured data. Accordingly, some corrections were made in multiple sections, including the addition of the reference information for the PACE trial in the reference list. The updated sections are given in this Correction, and the changes have been highlighted in bold typeface. The original article [1] has been corrected.

\begin{abstract}
The updated sentences are given below, and the changes have been highlighted in bold typeface.

Result: Among 513 potentially relevant articles, 56 RCTs met our inclusion criteria; these included 25 RCTs of 22 different pharmacological interventions, 29 RCTs of 19 non-pharmacological interventions and 2 RCTs of combined interventions. These studies accounted for a total of 6956 participants (1713 males and 5243 females, 6499 adults and 457 adolescents). CDC 1994 (Fukuda) criteria were mostly used for case definitions (42 RCTs, $\mathbf{7 5 . 0} \%$ ), and the primary measurement tools included
\end{abstract}

The original article can be found online at https://doi.org/10.1186/s1296 7-019-02196-9.

*Correspondence: ckson@dju.kr

${ }^{2}$ Institute of Traditional Medicine and Bioscience, Dunsan Oriental

Hospital of Daejeon University, 75, Daedeok-daero 176, Seo-gu, Daejeon, Republic of Korea

Full list of author information is available at the end of the article the Checklist Individual Strength (CIS, 35.7\%) and the 36-item Short Form health survey (SF-36, 32.1\%). Eight interventions showed statistical significance: 3 pharmacological (Staphypan Berna, Poly(I):poly(C12U) and CoQ10 + NADH) and 5 non-pharmacological therapies (cognitive-behavior-therapy-related treatments, gradedexercise-related therapies, rehabilitation, acupuncture and abdominal tuina). However, there was no definitely effective intervention with coherence and reproducibility.

\section{Result section}

The updated sentences are given below, and the changes have been highlighted in bold typeface.

\section{Characteristics of RCTs meeting the inclusion criteria}

From the PubMed and Cochran databases, a total of 513 articles were initially identified, and $\mathbf{5 6}$ articles ultimately met the inclusion criteria for this study (Fig. 1). Fifty-one RCTs $(\mathbf{9 1 . 1} \%)$ were conducted for adult patients, while 5 RCTs $(\mathbf{8 . 9} \%)$ were conducted for the adolescent population (Table 1). The majority of RCTs were conducted in 3 countries: the UK $(n=16)$, the Netherlands $(n=14)$, and the USA $(n=9)$. Regarding interventions, 29 RCTs $\mathbf{5 1 . 8} \%)$ conducted nonpharmacological interventions, 25 RCTs (44.6\%) conducted pharmacological interventions and 2 RCTs conducted a combination of pharmacological and nonpharmacological interventions (Tables 2 and 3).

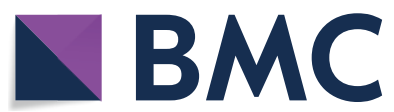

(c) The Author(s) 2020. This article is licensed under a Creative Commons Attribution 4.0 International License, which permits use, sharing, adaptation, distribution and reproduction in any medium or format, as long as you give appropriate credit to the original author(s) and the source, provide a link to the Creative Commons licence, and indicate if changes were made. The images or other third party material in this article are included in the article's Creative Commons licence, unless indicated otherwise in a credit line to the material. If material is not included in the article's Creative Commons licence and your intended use is not permitted by statutory regulation or exceeds the permitted use, you will need to obtain permission directly from the copyright holder. To view a copy of this licence, visit http://creativeco mmons.org/licenses/by/4.0/. The Creative Commons Public Domain Dedication waiver (http://creativecommons.org/publicdomain/ zero/1.0/) applies to the data made available in this article, unless otherwise stated in a credit line to the data. 


\section{Characteristics of participants and case definitions} for inclusion criteria

In 56 RCTs, a total of 6956 participants (1713 males and 5243 females, 6499 adults with a mean age of 40.2 \pm 4.0 years and 457 adolescents with a mean age of $15.5 \pm 0.3$ years) were enrolled. Fifty-five RCTs (98.2\%) adapted at least one of the following CFS case definitions: CDC 1994 (Fukuda) criteria (42 RCTs), Oxford 1991 (Sharpe) criteria (13 RCTs), CDC 1988 (Holmes) criteria (3 RCTs), Lloyd 1988 criteria (2 RCTs), and Schluederberg 1992 (2 RCTs).

\section{Main outcome measurement}

A total of 31 primary measurement tools were used to assess the main outcome in $\mathbf{5 6}$ RCTs. The Checklist Individual Strength (CIS) was the most frequently used (35.7\%), and others included the 36-item Short Form health survey (SF-36, 32.1\%), Sickness Impact Profile (SIP, 14.3\%), Chalder Fatigue Scale (14.3\%), Visual Analogue Scale (VAS, 10.7\%) and Clinical Global Impression (CGI, 8.9\%). There were 29 RCTs that used multiple primary measurements (Table 1 ).

\section{RCTs with nonpharmacological interventions}

There were 29 RCTs in the nonpharmacological category (26 for adults, 3 for adolescents) with 19 kinds of interventions, mainly CBT $(n=12)$, exercise $(n=6)$, and self-care $(n=5)$. The mean treatment period was $18.5 \pm 8.9$ weeks $(\mathbf{1 7 . 1} \pm 7.1$ weeks for adults, $30.7 \pm 15.1$ weeks for adolescents). Of the $\mathbf{1 2}$ CBT subcategories, 6 RCTs showed statistical effectiveness of CBT compared to the control $[41,44,46,49,50,52]$. In addition, 4 RCTs of graded-exercise-related therapies $[46,53,55,56]$ and 3 RCTs of integrative, consumer-driven rehabilitation [64], acupuncture [65] and abdominal tuina [67] showed a significantly effect of the intervention compared to the control (Table 3).

\section{Discussion section}

The updated sentences are given below, and the changes have been highlighted in bold typeface. Sentences with only a change in reference citations numbering (the original references 46-92 were re-numbered to 47-93) are not provided.

\section{The first paragraph (the 3rd sentence)}

To support future studies for CFS/ME treatments, we systematically reviewed 56 RCTs to investigate characteristics such as participants, case definitions, interventions and primary measurements.

\section{The second paragraph (the 1 st sentence)}

The sex ratio of the participants was male 1 vs. female 3 (1713/5143, except one RCT had recruited only females).

The third paragraph (the 1st-4th sentences)

A total of 56 RCTs included 25 pharmacological, 29 nonpharmacological and 2 combined interventions (Table 1 ). The mean treatment period of the RCTs with nonpharmacological interventions was longer than that with medication, especially for adolescents (total: $\mathbf{1 8 . 5} \pm \mathbf{8 . 9}$ vs. $10.8 \pm 6.8$, adolescent: $30.7 \pm 15.1$ vs. $8.5 \pm 0.7$, Table 1). Periodically, the trials gradually increased, with 13 trials in the 1990s, 19 trials in the 2000s and 24 trials in the 2010s. The pharmacological RCTs were predominant in the 1990s and 2000s, while nonpharmacological interventions became predominant in the 2010s (pharm acological:nonpharmacological ratio from 20:14 to 7:17) (data not shown).

\section{The fifth paragraph (the 8th and 11th sentences)}

Contrary to the positive outcomes in the 1990s and 2000s, more recent CBT trials have failed to show consistent benefits in patients with CFS/ME: 5 of 8 RCTs of CBT did not show significant effects in our data.

In our data, 5 of $\mathbf{6}$ RCTs with graded-exercise-related therapies presented positive outcomes; however, the clinical usefulness of GET is highly controversial [89].

\section{The eighth paragraph (the 5th sentence)}

In addition, only 9 of 56 RCTs had presented fragmentary data related to blood parameters.

\section{Reference section}

As one RCT (PACE trial) was added, its reference information [2] was included in the reference list as reference number 46. Accordingly, the original references 46-92 were re-numbered to 47-93.

\section{Figures}

Figures 1 and 2. 


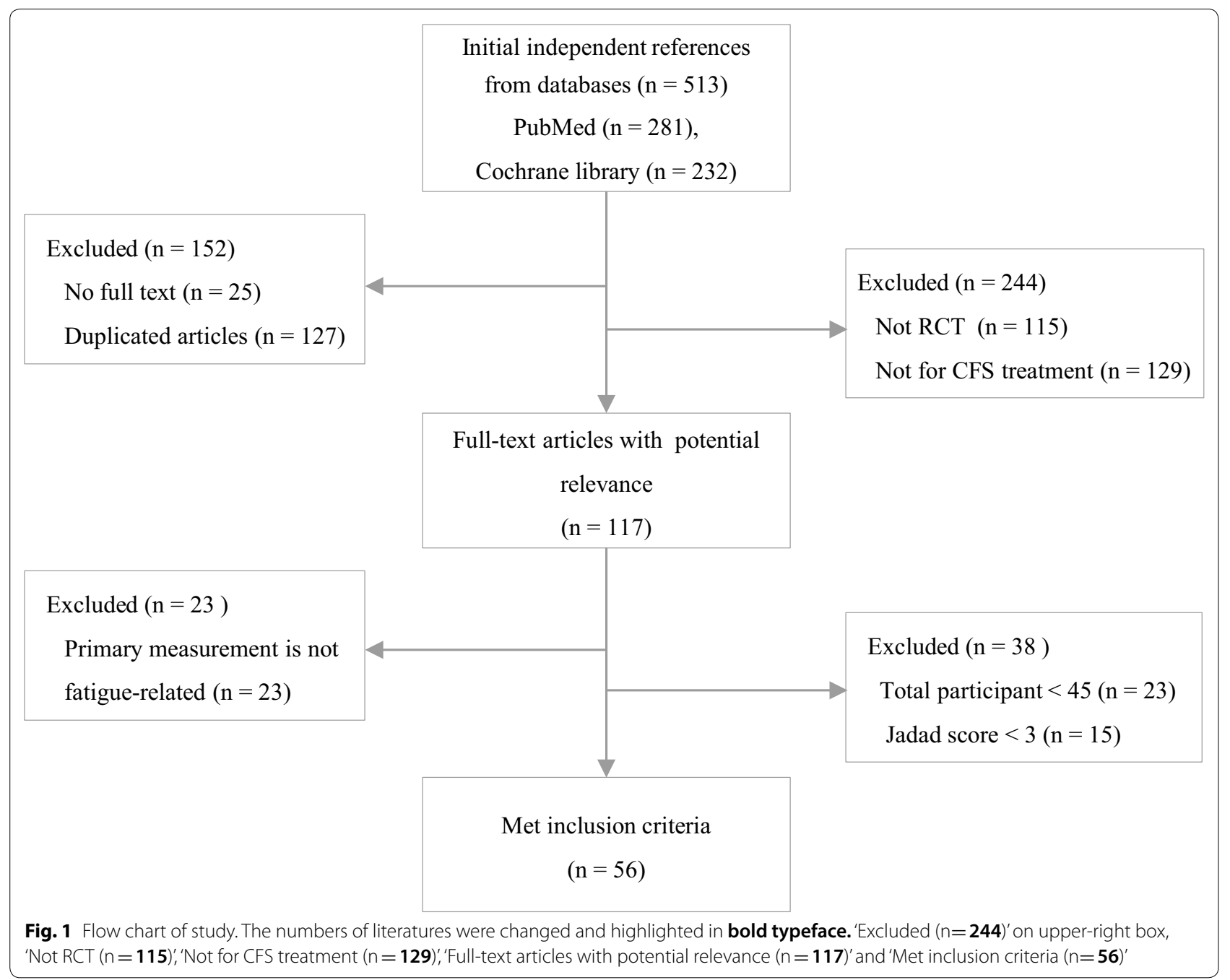




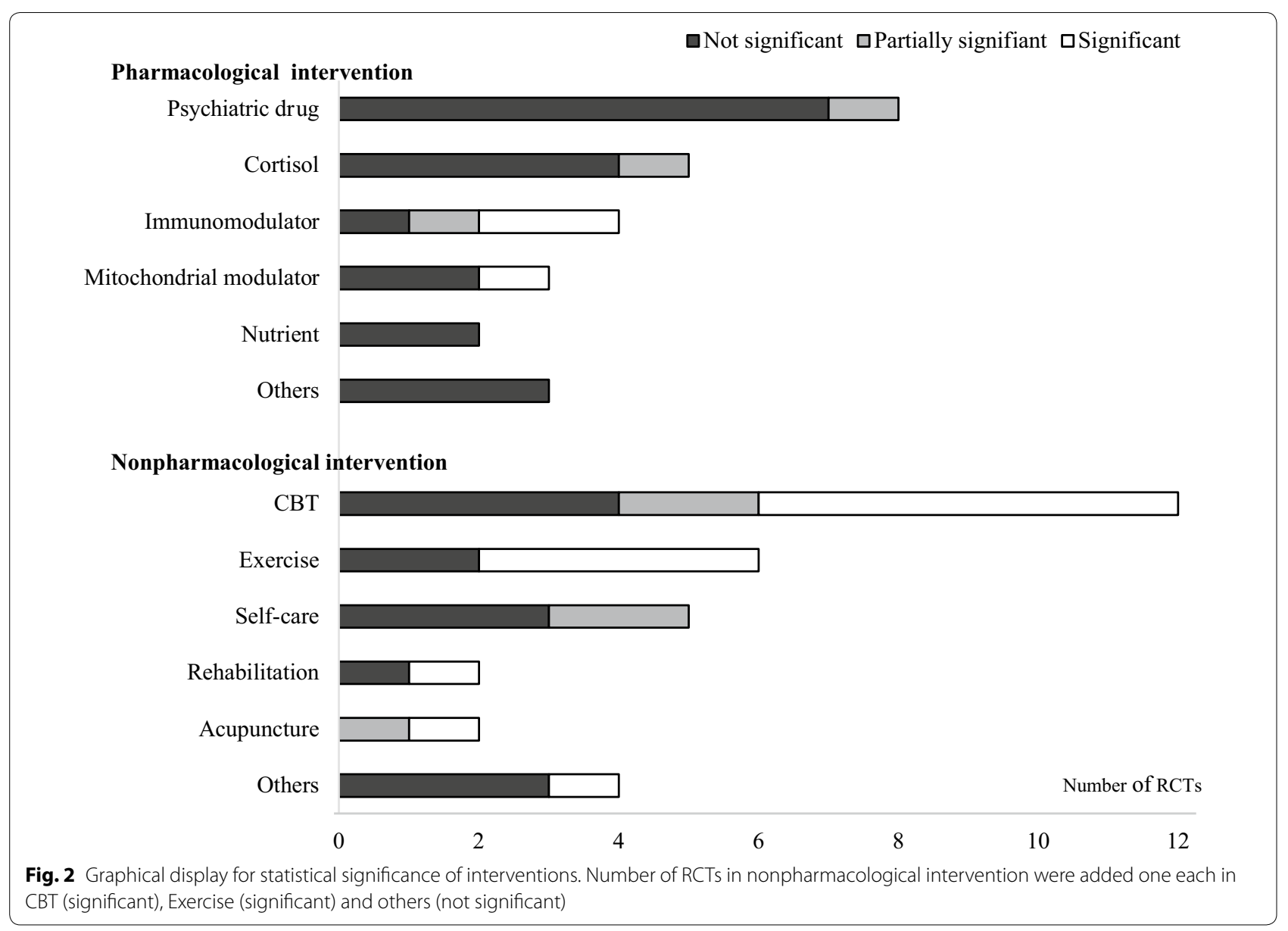




\section{Tables}

The updated Tables 1, 3 and 4 are given below, and the changes have been highlighted in bold typeface.

Table 1 Study characteristics

\begin{tabular}{|c|c|c|c|}
\hline Items & Adults & Adolescents & Total \\
\hline N. of RCT (\%) & $51(91.1)$ & $5(8.9)$ & $56(100.0)$ \\
\hline N. of participants (\%) (males/females) & $6499(93.4)(1611 / 4888)$ & $457(6.6)(102 / 355)$ & $6956(100.0)(1713 / 5243)$ \\
\hline Mean N. of participants & $127.4 \pm 113.3$ & $91.4 \pm 33.5$ & $124.2 \pm 109.0$ \\
\hline Mean age $(\text { year) })^{a}$ & $40.2 \pm 4.0$ & $15.5 \pm 0.3$ & $38.7 \pm 8.1$ \\
\hline \multicolumn{4}{|l|}{ N. of case definitions for inclusion criteria $(\%)^{b, c}$} \\
\hline CDC 1994 (Fukuda) & $37(72.5)$ & $5(100.0)$ & $42(75.0)$ \\
\hline Schluederberg 1992 & $2(3.9) 12(23.5)$ & - & $2(3.6) 13(23.2)$ \\
\hline Oxford 1991 (Sharpe) & $3(5.9)$ & $1(20.0)$ & $3(5.4)$ \\
\hline CDC 1988 (Holmes) & $2(3.9)$ & - & $2(3.6)$ \\
\hline Lloyd 1988 & $5(9.8)$ & - & $6(10.7)$ \\
\hline Others & & $1(20.0)$ & \\
\hline RCTs with pharmacological intervention (N, \%) & $23(92.0)$ & $2(8.0)$ & $25(100.0)$ \\
\hline Kinds of interventions (\%) & $20(90.9)$ & $2(9.1)$ & $22(100.0)$ \\
\hline Mean treatment period (weeks) & $11.0 \pm 7.0$ & $8.5 \pm 0.7$ & $10.8 \pm 6.8$ \\
\hline RCTs with nonpharmacological intervention ( $N, \%)$ & $26(89.7)$ & $3(10.3)$ & $29(100.0)$ \\
\hline Kinds of interventions $^{d}$ & $18(94.7)$ & $2(10.5)$ & $19(100.0)$ \\
\hline Mean treatment period (weeks) & $17.1 \pm 7.1$ & $30.7 \pm 15.1$ & $18.5 \pm 8.9$ \\
\hline RCTs with combined interventions ( $N, \%)$ & $2(100.0)$ & - & $2(100.0)$ \\
\hline Kinds of interventions (\%) & $4(100.0)$ & - & $4(100.0)$ \\
\hline Mean treatment period (weeks) & $26 \pm 2.8$ & - & $26 \pm 2.8$ \\
\hline \multicolumn{4}{|l|}{ Primary measurements in 55 RCTs $(n, \%)^{\text {ce }}$} \\
\hline Checklist Individual Strength (CIS) & & $20(35.7)$ & \\
\hline 36-item Short Form health survey (SF-36) & & $18(32.1)$ & \\
\hline Sickness Impact Profile (SIP) & & $8(14.3)$ & \\
\hline Chalder Fatigue Scale & & $8(14.3)$ & \\
\hline Visual Analogue Scale (VAS) & & $6(10.7)$ & \\
\hline Clinical Global Impression (CGI) & & $5(8.9)$ & \\
\hline Karnofsky Performance Scale (KPS) & & $3(5.4)$ & \\
\hline School attendance rate (SAR) & & $3(5.4)$ & \\
\hline Multidimensional Fatigue Inventory (MFI) & & $2(3.6)$ & \\
\hline Fatigue Severity Scale (FSS) & & $2(3.6)$ & \\
\hline Others & & $21(37.5)$ & \\
\hline
\end{tabular}

a This is the mean of ages presented as median or mean in original articles

b Twelve RCTs used two case definitions for inclusion criteria

c Some items have been applied multiple times, thus the total percentage is larger than $100 \%$

d One intervention (CBT) was used for both of adult and adolescent studies

e Twenty-nine RCTs used multiple primary measurements 
Table 3 RCTs with nonpharmacological interventions

\begin{tabular}{|c|c|c|c|c|}
\hline Intervention & $\begin{array}{l}\text { N. of participants (N. of arms, } \\
\text { control) }\end{array}$ & Period (week) & $\begin{array}{l}\text { Primary measurement } \\
\text { (subscale) }\end{array}$ & Significance \\
\hline \multicolumn{5}{|l|}{ CBT } \\
\hline iCBT [41] & 240 (3, waitlist) & 27 & CIS (fatigue) & $P<0.01$ \\
\hline Group CBT [42] & 204 (3, waitlist) & 24 & CIS (fatigue), SF-36 (physical score) & CIS: $d>0.8$ \\
\hline CBT [43] & $122(2, \mathrm{MRT})$ & 24 & CIS (fatigue), SF-36 & Not significant \\
\hline FITNET [44] & 135 (2, usual care) & 48 & $\begin{array}{l}\text { SAR, CIS (fatigue), CHQ (physical } \\
\text { score) }\end{array}$ & $P<0.01$ \\
\hline $\mathrm{CBT}+\mathrm{GET}[46]$ & 120 (2, usual care) & 24 & SF-36 & Not significant \\
\hline CBT $[46]$ & $640(4, M C)$ & 24 & $\begin{array}{l}\text { Chalder scale, SF-36 (physical } \\
\text { score) }\end{array}$ & $P<0.01$ \\
\hline Family-focused CBT [47] & 63 (2, psychoeducation) & 24 & SAR & Not significant \\
\hline Group CBT [48] & 153 (3, education + support, MC) & 16 & SF-36 (physical, mental score) & Not significant \\
\hline CBT [49] & 71 (2, waitlist) & 20 & $\begin{array}{l}\text { CIS (fatigue), SF-36 (physical } \\
\text { score), SAR }\end{array}$ & CIS, SF-36: $P<0.01, S A R: P<0.05$ \\
\hline CBT $[\mathbf{5 0}]$ & $\begin{array}{l}278 \text { ( } 3 \text {, guided support, no treat- } \\
\text { ment) }\end{array}$ & 32 & CIS (fatigue), SIP-8 & CIS: $P<0.01$, SIP: $P<0.05$ \\
\hline CBT $[\mathbf{5 1}]$ & $60(2$, relaxation $)$ & $16-24$ & $\begin{array}{l}\text { Chalder scale, SF-36 (physical } \\
\text { score) }\end{array}$ & Chalder scale: $P<0.01$ \\
\hline CBT [52] & $60(2, M C)$ & 16 & Karnofsky normal function scale & $P<0.01$ \\
\hline \multicolumn{5}{|l|}{ Exercise } \\
\hline Guided exercise self-help [53] & $211(2, M C)$ & 12 & $\begin{array}{l}\text { Chalder scale, SF-36 (physical } \\
\text { score) }\end{array}$ & $P<0.01$ \\
\hline Qigong [54] & 64 (2, waitlist) & 16 & Chalder scale, SF-12 & Not significant \\
\hline GET [46] & $640(4, M C)$ & 24 & $\begin{array}{l}\text { Chalder scale, SF-36 (physical } \\
\text { score) }\end{array}$ & $P<0.01$ \\
\hline GET [55] & $49(2, M C)$ & 12 & Self-rated global change score & $P<0.05$ \\
\hline $\begin{array}{l}\text { Education to encourage graded } \\
\text { exercise [56] }\end{array}$ & $148(4, M C)$ & 16 & SF-36 (physical score) & $P<0.01$ \\
\hline Graded aerobic exercise [57] & 66 (crossover, flexibility therapy) & 12 & CGl & Not significant \\
\hline \multicolumn{5}{|l|}{ Self-care } \\
\hline Fatigue self-management [58] & 137 (3, usual care) & 12 & FSS & Not significant \\
\hline $\begin{array}{l}\text { Group-based self-management } \\
\text { [59] }\end{array}$ & 137 (2, usual care) & 16 & SF-36 (physical score) & Not significant \\
\hline Guided self-instruction [60] & 123 (2, waitlist) & 20 & $\begin{array}{l}\text { CIS (fatigue), SF-36 (physical, social } \\
\text { score) }\end{array}$ & CIS: $P<0.01$ \\
\hline Stepped care [61] & $171(2, \mathrm{CBT})$ & 16 & $\begin{array}{l}\text { CIS (fatigue), SIP-8, SF-36 (physical } \\
\text { score) }\end{array}$ & Not significant \\
\hline Guided self-instruction [62] & 169 (2, waitlist) & 16 & $\begin{array}{l}\text { CIS (fatigue), SIP-8, SF-36 (physical } \\
\text { score) }\end{array}$ & CIS, SIP8: $P<0.01$ \\
\hline \multicolumn{5}{|l|}{ Rehabilitation } \\
\hline Pragmatic rehabilitation [63] & $\begin{array}{l}302 \text { ( } 3 \text {, supportive listening, gen- } \\
\text { eral treatment) }\end{array}$ & 18 & $\begin{array}{l}\text { Chalder scale, SF-36 (physical } \\
\text { score) }\end{array}$ & Not significant \\
\hline $\begin{array}{l}\text { Integrative, consumer-driven } \\
\text { rehabilitation [64] }\end{array}$ & 47 (2, delayed program) & 16 & $\begin{array}{l}\text { CFS Symptom Rating Form, The } \\
\text { QoL Index }\end{array}$ & $P<0.05$ \\
\hline \multicolumn{5}{|l|}{ Acupuncture } \\
\hline Acupuncture [65] & 150 (3, sa-am, no treat) & & FSS & $P<0.05$ \\
\hline Acupuncture [66] & $100(2$, sham $)$ & & $\begin{array}{l}\text { Chalder scale, SF-12, GHQ-12 } \\
\text { (mental score) }\end{array}$ & Chalder scale: $P<0.05$ \\
\hline
\end{tabular}


Table 3 (continued)

\begin{tabular}{|c|c|c|c|c|}
\hline Intervention & $\begin{array}{l}\text { N. of participants (N. of arms, } \\
\text { control) }\end{array}$ & Period (week) & $\begin{array}{l}\text { Primary measurement } \\
\text { (subscale) }\end{array}$ & Significance \\
\hline \multicolumn{5}{|l|}{ Others } \\
\hline Abdominal tuina [67] & 77 (2, acupuncture) & & Chalder scale, SAS, HAMD & $P<0.05$ \\
\hline Adaptive pacing $[46]$ & $640(4, M C)$ & 24 & $\begin{array}{l}\text { Chalder scale, SF-36 (physical } \\
\text { score) }\end{array}$ & Not significant \\
\hline Low-sugar, low-yeast diet [68] & $52(2$, healthy eating $)$ & 24 & Chalder scale, SF-36 & Not significant \\
\hline Distant healing [69] & 409 (4, not knowing, no treat) & 24 & SF-36 (mental score) & Not significant \\
\hline
\end{tabular}

CBT cognitive behavior therapy, FITNET: Fatigue In Teenagers on the interNET, GET graded exercise therapy, CIS Checklist Individual Strength, SF-36 36-item Short Form health survey, SAR school attendance rate, CHQ Child Health Questionnaire, SIP-8 Sickness Impact Profile, CGI Clinical Global Impression, FSS Fatigue Severity Scale, GHQ-12 General Health Questionnaire-12, SAS Self-rating Anxiety Scale, HAMD Hamilton rating scale for Depression

Table 4 RCTs with pharmacological and nonpharmacological combined interventions

\begin{tabular}{|c|c|c|c|c|}
\hline Intervention & Intervention & Intervention & Intervention & Intervention \\
\hline $\begin{array}{l}\text { Fluoxetine }+ \text { graded } \\
\text { exercise }[\mathbf{7 0}]\end{array}$ & $\begin{array}{l}\text { Exercise + fluoxetine: } 33 \\
\text { Exercise + placebo: } 34 \\
\text { Appointment + fluoxetine: } 35 \\
\text { Appointment + placebo: } 34\end{array}$ & $\begin{array}{l}24 \\
20 \mathrm{mg} / \mathrm{day}\end{array}$ & Chalder scale & $\begin{array}{l}\text { Graded exercise } \\
P<0.05\end{array}$ \\
\hline $\begin{array}{l}\text { Dialyzable leukocyte } \\
\text { extract (DLE) +CBT } \\
\text { [71] }\end{array}$ & $\begin{array}{l}\text { DLE + CBT: } 20 \\
\text { DLE + clinic: } 26 \\
\text { Placebo + CBT: } 21 \\
\text { Placebo + clinic: } 23\end{array}$ & $\begin{array}{l}28 \\
5 \times 10^{8} \text { leukocytes } \\
8 \text { times biweekly }\end{array}$ & VAS (global well-being) & Not significant \\
\hline
\end{tabular}

VAS Visual Analogue Scale

\section{Author details}

1 Korean Medical College of Daejeon University, 62, Daehak-ro, Dong-gu, Daejeon, Republic of Korea. ${ }^{2}$ Institute of Traditional Medicine and Bioscience, Dunsan Oriental Hospital of Daejeon University, 75, Daedeok-daero 176, Seo-gu, Daejeon, Republic of Korea.

Published online: 23 December 2020

\section{References}

1. Kim Do-Young, Lee Jin-Seok, Park Samuel-Young, Kim Soo-Jin, Son

Chang-Gue. Systematic review of randomized controlled trials for chronic fatigue syndrome/myalgic encephalomyelitis (CFS/ME). J Transl Med. 2020;18:7. https://doi.org/10.1186/s12967-019-02196-9.
2. White PD, Goldsmith KA, Johnson AL, Potts L, Walwyn R, DeCesare JC, Baber HL, Burgess M, Clark LV, Cox DL, Bavinton J, Angus BJ, Murphy G, Murphy M, O'Dowd H, Wilks D, McCrone P, Chalder T, Sharpe M. Comparison of adaptive pacing therapy, cognitive behaviour therapy, graded exercise therapy, and specialist medical care for chronic fatigue syndrome (PACE): a randomised trial. Lancet. 2011;377(9768):823-36. https://doi.org/10.1016/s0140-6736(11)60096-2 (Epub 2011 Feb 18).

\section{Publisher's Note}

Springer Nature remains neutral with regard to jurisdictional claims in published maps and institutional affiliations. 